\section{Why a One Size Fits All Approach to RAS Might Not Fit Colorectal Cancer}

\author{
Jonathan M. Loree, MD, and Scott Kopetz, MD, PhD
}

Since the discovery that KRAS exon 2 mutations predict a lack of benefit from antiepidermal growth factor receptor (EGFR) therapy in metastatic colorectal cancer, our understanding of RAS mutations has expanded considerably. Guidelines now suggest extended RAS characterization, which includes assessment of KRAS and NRAS for mutations at exon 2 (codons 12 and 13), exon 3 (codons 59 and 61), and exon 4 (codons 117 and 146). ${ }^{1}$ Extended RAS mutations appear to confer resistance to antiEGFR therapy. With their inclusion, approximately $56 \%$ of patients will be defined as having a RAS mutation and will not be eligible for anti-EGFR therapy. ${ }^{2,3}$ In addition, atypical RAS mutations exist that are of unclear significance and difficult to study in any prospective manner given their rarity. Even within guideline-described codons there are mutations, such as KRAS A59T, that are of unclear significance. Studying these mutations individually would be nearly impossible, and they highlight a pressing issue of how we deal with variants of unknown significance (VUS). The number of uncommon VUS is likely to expand exponentially as clinical sequencing moves to more comprehensive sequencing platforms, and we will need solutions to deal with these variants.

In this issue of JNCCN, Lou et al ("Therapeutic Response of Metastatic Colorectal Cancer Harboring a KRAS Missense Mutation After Combination Chemotherapy With the EGFR Inhibitor Panitumumab") present the case of a 50-year-old woman with a KRAS A59T-mutated metastatic colon cancer who received 4 cycles of FOLFIRI plus panitumumab and showed a radiographic partial response accompanied by a decline in carcinoembryonic antigen. The patient subsequently experienced disease control lasting 8 months while receiving FOLFIRI plus panitumumab. She was noted to have a KRAS A59T mutation after the treating institution changed from a Sanger sequencing platform specific to KRAS exon 2 to a more comprehensive amplicon-based next-generation sequencing (NGS) panel that included extended RAS coverage. The NGS panel was performed on DNA extracted from the same biopsy of the primary tumor as the Sanger sequencing. The authors propose that patients with KRAS A59T mutations may represent a population with continued sensitivity to anti-EGFR therapy.

\section{Challenges and Opportunities in Studying Rare Biomarkers}

Given the infrequency of many extended RAS mutations, they have not been well described individually and were considered as a group of mutations in the studies that support extended RAS mutation testing. Codon 59 mutations are particularly lacking in evidence to support their role in predicting resistance to anti-EGFR therapy. Only 7 patients with codon 59 mutations were described in the landmark PRIME trial analysis of extended RAS mutations, and these mutations were not part of the extended RAS mutation analysis. The evidence to support these mutations as a predictive marker was only the fact that removing these patients from the population with wild-type RAS resulted in a hazard ratio that made anti-EGFR agents appear more beneficial in wild-type patients. ${ }^{3}$

Clearly, further characterization of these variants is required, but how do we characterized them in a meaningful way? Using in vitro assays is one option. At our institution, the Precision Oncology Decision Support Team has a functional genomics assay using $\mathrm{BaF} 3$ and $\mathrm{MCF} 10 \mathrm{~A}$ cell viability studies to help characterize unknown

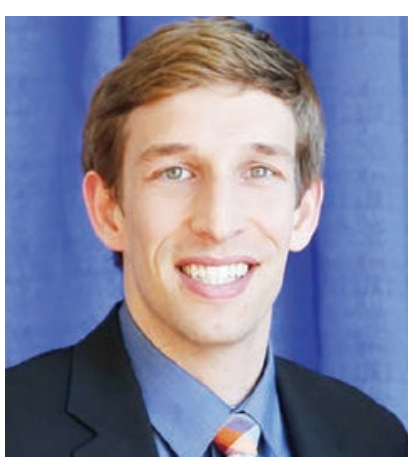

Jonathan Loree, MD

Jonathan Loree, MD, is a research fellow at The University of Texas MD Anderson Cancer Center, where his research interests include tumor heterogeneity and biomarkers in colorectal cancer. He completed his medical degree and internal medicine training at the University of Alberta, after which he completed a medical oncology fellowship at the British Columbia Cancer Agency. He is the recipient of a J. Edward Mahoney ASCO Young Investigator Award, a Canadian Association of Medical Oncologists Fellowship Award, and a Royal College of Physicians and Surgeons of Canada Detweiler Travelling Fellowship, and is a member of the University of British Columbia Clinician Investigator Program. He is currently enrolled in a Master's degree program from MD Anderson UTHealth GSBS during his research fellowship at MD Anderson. 


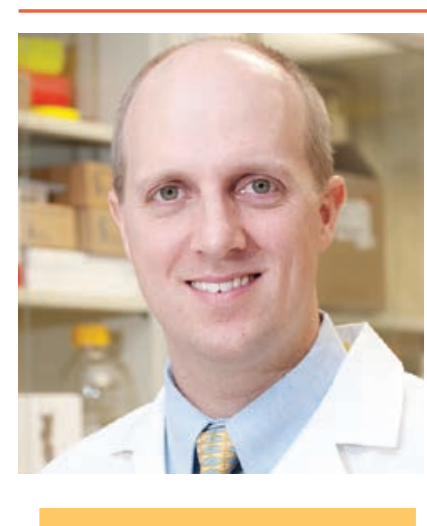

Scott Kopetz, MD, PhD

Scott Kopetz, MD, PhD, is an Associate Professor and the Deputy Chair of Translational Research for the Department of Gastrointestinal Medical Oncology at The University of Texas MD Anderson Cancer Center. He graduated summa cum laude from Vanderbilt University with a bachelor's degree in biomedical engineering/electrical and received his medical degree from Johns Hopkins School of Medicine. He obtained his residency training in internal medicine at Duke University Medical Center, followed by a medical oncology fellowship and $\mathrm{PhD}$ at MD Anderson Cancer Center. He has authored more than 100 peer-reviewed articles, is a senior editor for Clinical Cancer Research, and is an editorial board member on Journal of Clinical Oncology and JNCI. He is vice-chair for colon cancer research in the NSABP/RTOG cooperative group, and a member of the NIH Gastrointestinal Oncology Steering Committee. $\mathrm{He}$ is the principal investigator for several phase I and II clinical trials, including of Assessment of Targeted Therapies Against Colorectal Cancer (ATTACC) study, a novel biomarker enrichment program for colorectal cancer. His research interests include the biology of the refractory colorectal cancer and the development of novel therapeutics for molecularly distinct subsets of colorectal cancer patients. mutations, but is this enough information to guide patient care? Another challenge with functional assays is that these techniques are not available to guide decisions outside of a specialized center, making them impractical for most clinical care.

A different approach to studying rare variants is the potential to incorporate real-world evidence. As we move into an era of electronic health records and big data, the ability to draw information from large data sets that are pooled between multiple institutions represents a unique opportunity to study uncommon situations. By harnessing the strengths of studying entire populations rather than just focusing on patients enrolled on a trial, we may be able to answer difficult questions. For example, there are 8 patients with KRAS A59T mutations noted in the COSMIC database; however, our own institution's database notes another $5 .{ }^{4}$ Other institutions may also have information on a handful of these patients. If the outcomes data on all of these patients were merged, we may be able to make a meaningful statement about the particular mutation.

These efforts are complicated by privacy concerns, administrative hurdles to merging data from different health authorities, and the need for standardized reporting to facilitate pooled analyses. Despite these challenges, there is a growing realization that real-world data are useful. In fact, the FDA is in the process of drafting guidelines to suggest how premarketing and postmarketing regulatory requirements may be addressed using real-world evidence to complement traditional clinical trials. Efforts such as The Cancer Genome Atlas and the American Association for Cancer Research (AACR) Project Genie are working to bring together genomic repositories and clinical annotation in platforms that can be used to ask questions regarding biomarkers. These data sets not only allow studies in large populations but also make us more efficient as a research community.

Context of a variant and biologic rationale may also help guide us in our efforts to understand the clinical significance of uncommon alterations. Many of the extended RAS variants in exon 3 and 4 are more commonly detected in patients who have previously been treated with anti-EGFR therapy and were originally categorized as RAS wild-type. ${ }^{6}$ The fact that these alterations arose under selective pressure and coincide with clinical progression strongly argues that they predict resistance to antiEGFR therapies and supports extended RAS mutations as predictive biomarkers. Unfortunately, KRAS codon 59 mutations have not been described as a mechanism of resistance in colorectal cancer to help guide our assessment of the current case.

\section{Is KRAS A59T Truly Sensitive to Anti-EGFR Therapy?}

Although reports of patients with exceptional response can provide insight into the biology underlying response, application to clinical decision-making needs to be tempered. When we see reports of a single patient with a genomic biomarker experiencing an exceptional outcome, we must always consider whether a sequencing error could be present. Tumor heterogeneity is another important consideration not mentioned by the authors that should be considered when targeted therapies result in unexpected outcomes. Concordance between primary tumor and liver metastases RAS status appears high in colorectal cancer, ranging from $90 \%$ to $100 \%$; however, discordance of up to $32 \%$ has been reported between lung or lymph node metastases and primary tumors. ${ }^{7,8}$ In the reported case, a partial response was noted in the pulmonary metastases, although whether the liver or primary tumor responded to therapy is unclear. Given that the primary tumor is where the KRAS mutant clone was sampled, this information is particularly important. It is possible that the partial response was noted only in KRAS wild-type clonal populations, and that this exceptional response is not so exceptional. 
A major barrier to understanding clonal dynamics is that we cannot truly know the genotype of every cell within a patient at all times. Possibly, patients with only part of their total tumor mass containing a RAS mutation may still derive benefit from anti-EGFR therapy. In the CRYSTAL trial, low allele frequency RAS mutations identified using highly sensitive bead emulsion PCR were shown to predict a lack of benefit in overall survival from anti-EGFR therapy. ${ }^{9}$ However, objective response rates appeared to show trends toward benefit in the low mutant allele frequency group. The strength of this trend continuously increased as the mutant allele proportion decreased. In the current report, we lack information about the mutant allele frequency and the mutation status of the metastases that responded to therapy, and this may be an important driver of response.

\section{Conclusions}

In the right context, KRAS A59T mutations may be sensitive to anti-EGFR agents. Does this case report prove it? No. It raises an important question about whether all RAS mutations are created equally and highlights the difficulties in characterizing uncommon variants. Although in vitro assays and real-world evidence may help us understand the significance of these variants, they are not definitive. A recent review of the literature and internal databases at our institution noted 98 unique RAS variants, many of which lacked characterization. We will never be able to fully define the significance of all of these mutations with prospectively collected data, and some degree of uncertainty will always remain. However, guidelines are built on best available evidence, and it will require more than case reports to justify a change.

\section{References}

1. Sepulveda AR, Hamilton SR, Allegra CJ, et al. Molecular biomarkers for the evaluation of colorectal cancer: guideline from the American Society for Clinical Pathology, College of American Pathologists, Association for Molecular Pathology, and the American Society of Clinical Oncology [published online ahead of print February 6 , 2017]. J Clin Oncol, doi: 10.1200/JCO.2016.71.9807.

2. Peeters M, Kafatos G, Taylor A, et al. Prevalence of RAS mutations and individual variation patterns among patients with metastatic colorectal cancer: a pooled analysis of randomised controlled trials. Eur J Cancer 2015;51:1704-1713.

3. Douillard JY, Oliner KS, Siena S, et al. Panitumumab-FOLFOX4 treatment and RAS mutations in colorectal cancer. N Engl J Med 2013;369:1023-1034.

4. Forbes SA, Beare D, Gunasekaran P, et al. COSMIC: exploring the world's knowledge of somatic mutations in human cancer. Nucleic Acids Res 2015;43:D805-811.

5. Sherman RE, Andersen SA, Dal Pan GJ, et al. Real-world evidence-what is it and what can it tell us? N Engl J Med 2016;375:2293-2297.

6. Morelli MP, Overman MJ, Dasari A, et al. Characterizing the patterns of clonal selection in circulating tumor DNA from patients with colorectal cancer refractory to anti-EGFR treatment. Ann Oncol 2015;26:731-736.

7. Han CB, Li F, Ma JT, Zou HW. Concordant KRAS mutations in primary and metastatic colorectal cancer tissue specimens: a meta-analysis and systematic review. Cancer Invest 2012;30:741-747.

8. Baas JM, Krens LL, Guchelaar HJ, et al. Concordance of predictive markers for EGFR inhibitors in primary tumors and metastases in colorectal cancer: a review. Oncologist 2011;16:1239-1249.

9. Van Cutsem E, Lenz HJ, Kohne CH, et al. Fluorouracil, leucovorin, and irinotecan plus cetuximab treatment and RAS mutations in colorectal cancer. J Clin Oncol 2015;33:692-700. 